\title{
Disturbed Sleep and Disturbed Bowel Functions: Implications for Constipation in Healthy Individuals
}

\author{
Kok-Ann Gwee \\ Yong Loo Lin School of Medicine, National University of Singapore and Gleneagles Hospital, Singapore
}

Article: Impact of sleep dysfunction on anorectal motility in healthy humans

Liu TT, Yi CH, Chen CL, Orr WC

(J Neurogastroenterol Motil 2011;17:180-184)

In 1993, Goldsmith and Levin brought to our attention that disturbed sleep could have an adverse effect on bowel functions. ${ }^{1}$ They employed a diary to record the relationship between sleep disturbance and bowel symptoms in subjects with irritable bowel syndrome (IBS). They found that morning IBS symptoms were increased when the quality of sleep was poor the night before, and decreased when subjects had slept better. These findings were replicated by a study of women with IBS which found that sleep disturbance one night predicted gastrointestinal symptoms the following day. ${ }^{2}$ Neither study analysed individual bowel symptoms. While disruption of biologic rhythms secondary to night shift work has been associated with gastrointestinal symptoms, these studies have not specifically examined the effects on bowel habits. ${ }^{3}$ To study the effects of disturbed sleep on bowel functions, we recruited healthy female full time nurses working in a tertiary hospital to keep a bowel and sleep diary for 2 weeks. Of 60 nurses working regular hours during the day only, we found constipation symptoms in 8 (13\%), while in 58 nurses working on rotating shifts involving working 4-8 night shifts per month, we found constipation symptoms in $14(24 \%))^{4}$ Using multivariate analysis, we showed that sleep disturbance, decreased well-being, anxiety, somatic pain and age were all independent predictors of bowel disturbance. Based on these observations, we hypothesized that disturbed sleep could affect bowel functions in healthy individuals.

In this journal, Liu et al, from Taiwan, have studied anorectal physiology in university students, comparing those with good and poor sleep quality as measured by the Pittsburgh Sleep Quality Index (PSQI) score. In this cohort, IBS was rigorously excluded by applying the Rome III criteria. In addition, subjects with fibromyalgia were also excluded. Thus, the focus of this study is on defecation function, rather than on visceral or somatic pain perception. Not surprisingly, they did not find any differences in sensitivity to rectal distension between good and poor sleepers. Rather, they found that PSQI scores correlated positively with rectal compliance. What this means is that the poorer the sleep,

Received: March 19, 2011 Revised: March 24, 2011 Accepted: March 24, 2011

(c) This is an Open Access article distributed under the terms of the Creative Commons Attribution Non-Commercial License (http://creativecommons. org/licenses/by-nc/3.0) which permits unrestricted non-commercial use, distribution, and reproduction in any medium, provided the original work is properly cited.

*Correspondence: Kok-Ann Gwee, FRCP, PhD

Associate Professor, Gleneagles Hospital, Annexe Block 05-37, 6A Napier Road, Singapore 258500

Financial support: None.

Tel: +65-6-474-6848, Fax: +65-6-475-8285, E-mail: slbclinic@gmail.com

Conflicts of interest: None. 
the greater the rectal compliance. Rectal compliance is the measure of the capacity of the rectal wall to stretch upon pressure stimulus. Increased rectal compliance has been proposed to be a contributing mechanism underlying constipation. The implication is that poor sleep may predispose a previously healthy individual to constipation. Sleep disturbance as a potential pathogenic factor in constipation has not been previously explored.

The limitation of the present study was that the assessment of sleep quality was retrospective. In order to explore the role of sleep disturbance in constipation, we would need to study in a prospective manner the effects of sleep deprivation on anorectal physiology, and correlate these changes with the quality of defecation. This approach was employed by Bergin and Read; 8 healthy male volunteers were confined in the investigation unit and kept awake for 35 hours, while an equal number were allowed to return home to sleep overnight. ${ }^{5}$ Anorectal manometry and rectal distension studies were carried out at the start of the study, and repeated after 24 hours, in both groups. In the sleep deprived group, sensitivity to rapid rectal distension stimulus increased, while in the control group, no significant change was observed. This observation suggests that sleep deprivation could predispose to visceral hypersensitivity which has been implicated as a pathogenic mechanism for abdominal pain in IBS. Unfortunately, this study did not examine in detail the effects of sleep on constipation. The authors reported no differences in the number of bowel movements between the 2 groups, but the changes in stool consistency and feeling of incomplete evacuation were not assessed. However, if we examine their data carefully, in their sleep deprived group there was actually a trend for an increase in rectal compliance that was not discerned in the control group.

Irregular working hours is no longer just a fact of life for doctors and nurses. With globalisation, workers in more and more industries will have to accept work schedules that will result in shorter sleep duration and a change to their usual sleep routine. Thus, it is important that more, and better planned, studies are conducted to define the effects of sleep disturbances and chronobiological factors on bowel functions.

\section{References}

1. Goldsmith G, Levin JS. Effect of sleep quality on symptoms of irritable bowel syndrome. Dig Dis Sci 1993;38:1809-1814.

2. Jarrett M, Heitkemper M, Cain KC, Burr RL, Hertig V. Sleep disturbance influences gastrointestinal symptoms in women with irritable bowel syndrome. Dig Dis Sci 2000;45:952-959.

3. Knutsson A. Health disorders of shift workers. Occup Med (Lond) 2003;53:103-108.

4. Zhen Lu W, Ann Gwee K, Yu Ho K. Functional bowel disorders in rotating shift nurses may be related to sleep disturbances. Eur J Gastroenterol Hepatol 2006;18:623-627.

5. Bergin AJ, Read NW. The effects of sleep deprivation on rectal sensitivity in healthy volunteers. Eur J Gastroenterol Hepatol 1993;5:527532 . 